\title{
Grid-connected photovoltaic module and array sizing based on an iterative approach
}

\author{
F. Q. Alenezi ${ }^{*}$ J. K. Sykulski, M. Rotaru \\ Electronics and Computer Science, University of Southampton, UK
}

\begin{abstract}
This paper deals with the sizing of a simple but efficient grid-connected photovoltaic (GCPV) system to minimize the difference between the electrical energy generated by the GCPV system and the required energy. It provides a technical sizing procedure based on an iterative approach for meeting specific amount of GWh output required by a future PV system considered for Kuwait. The iterative sizing algorithm has been implemented to determine the configuration of the PV array, actual rated output power with its relative energy, area of the array and efficiency of the system using an existing PV module and inverter database. Consequently the size of the array has been estimated based on a particular PV module and inverter combination. Optimum inverter-to-PV array sizing factor for a gridconnected PV system was determined in terms of the total system output.
\end{abstract}

Keywords: Photovoltaic module, standard test condition (STC), grid-connected photovoltaic (GCPV), inverter

\section{Introduction}

One of the primary concerns in designing any PV system is the determination of the correct size of the PV array or panel to supply a required unit of energy at a specified reliability [1-3]. It is appropriate to use monthly and annually averaged solar radiations (SR) to determine array capacity because of the influence of the dynamic behaviour of the PV system and the stochastic nature of SR on the required array capacity. Therefore, it is normal that the design should be done on meteorological data, SR and on the exact load profile of consumers over long periods. It is very important to be able to predict as accurately as possible the annual energy delivered by the system in order to decide whether it makes economic sense [4]. Other specifications, such as voltages, currents, etc, need to be satisfied as well, but the monthly and annually averaged SR provides a good starting point to GCPV system sizing. The sizing process is to meet a desired amount of output energy in $\mathrm{kWh}$ required from the GCPV system and its objective is to minimize the difference between the electrical energy generated by the GCPV system and the required energy.

The sizing process is based on a manual approach. This approach is based on an iterative algorithm and is called sizing algorithm using iterative manual approach (SAIMA). Using this approach, a system designer will select a PV module and an inverter from a list of PV modules and inverters before trying to match the PV module and inverter characteristics. The algorithm then computes the PV panel or array size with its configuration, as well as the electrical data of the prospective system. If the PV panel or array matches the inverter characteristics, the sizing procedure has reached its optimal configuration. Otherwise, the same procedure is repeated after selecting another combination of a PV module and an inverter.

The SAIMA algorithm has been implemented in MATLAB for convenience. Fig. 1 shows the main stages of the procedure to satisfy the conditions of SAIMA. The most frequently asked questions in the sizing process are:

- How many $\mathrm{kWh} / \mathrm{yr}$ are required?

\footnotetext{
* Manuscript received July 10, 2013; revised September 13, 2013.

Corresponding author. E-mail address: fgae1c10@ecs.soton.ac.uk.
} 


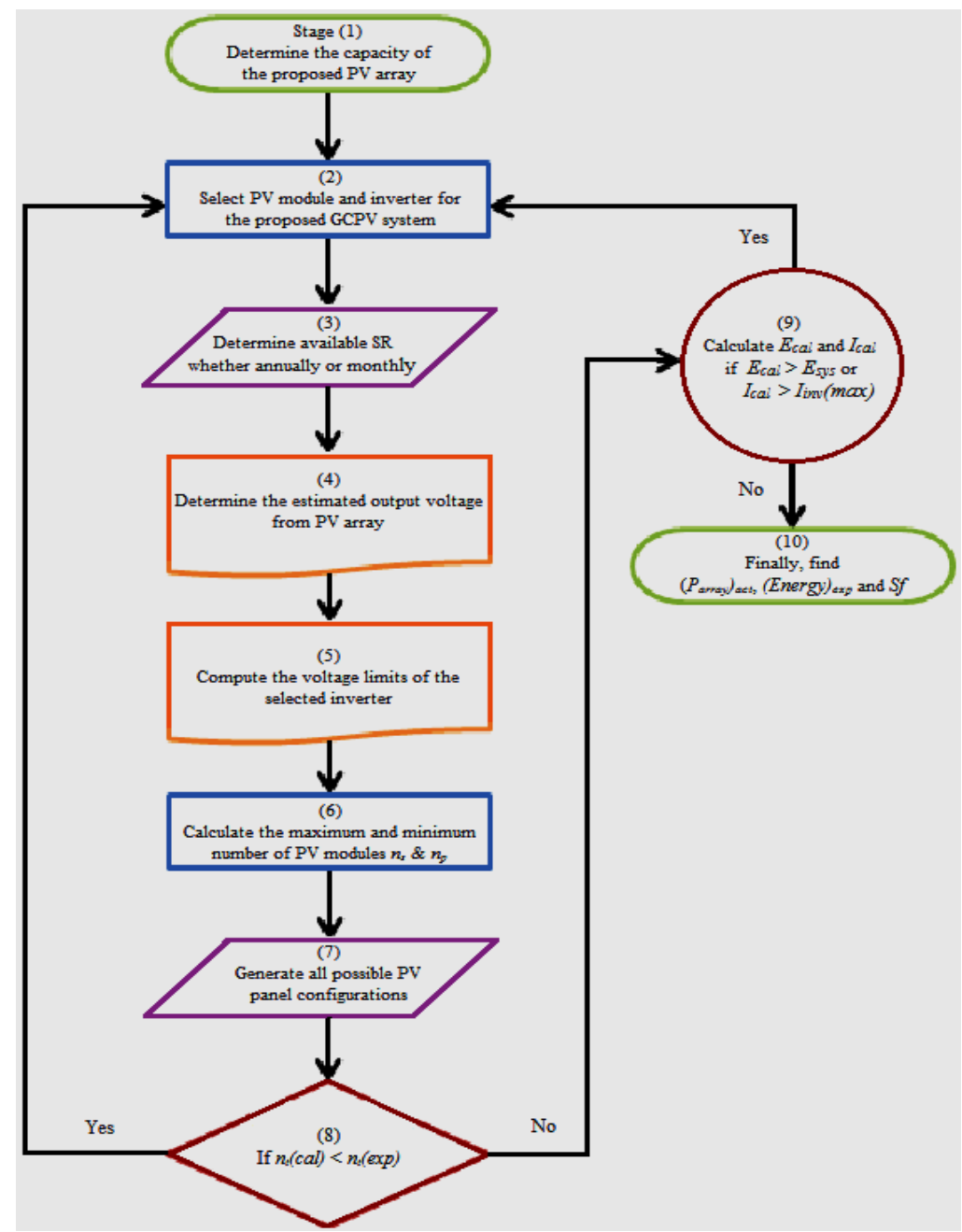

Fig. 1. Flowchart of sizing the proposed GCPV system.

- How many peak watts of dc PV power are needed to provide that amount?

- How much area will that system require?

A practical design, however, must include real components, which are available only in certain sizes and which have their own design constraints.

\section{Calculation of PV Outputs}

The electrical energy produced by a PV array is estimated using data from the monthly averaged SR on the horizontal plane, ambient temperature and the electrical data sheet of the PV module given by the manufacturer. These ratings are also used as inputs to the sizing process. The first step of SAIMA is to estimate the rated power, $\left(P_{\text {array }}\right)_{\mathrm{dc}}$, of the proposed PV array taking into consideration the following constraints:

- All the PV system is designed under standard test conditions (STC).

- A PV module and an inverter may only be selected from a specified list for the proposed GCPV system (see Table 1).

The electrical energy required, (Energy) $(\mathrm{GWh} / \mathrm{yr})$, will normally be specified by the investor; in our case,

$(\text { Energy })_{r}=200 \mathrm{GWh} / \mathrm{yr}$ 
Table 1. Required PV ratings for sizing algorithm

\begin{tabular}{ll}
\hline \hline \multicolumn{1}{c}{ Mequired ratings for the PV combination } \\
\hline \multicolumn{1}{c}{ Module } & \multicolumn{1}{c}{ Inverter } \\
\hline Open-circuit voltage, $E_{\mathrm{oc}}$ in volts & Maximum input voltage, $E_{\text {inv }}(\max )$ in volts \\
Short-circuit current, $I_{\mathrm{sc}}$ in amps & Minimum input voltage, $E_{\text {inv }}(\min )$ in volts \\
Maximum system voltage, $E_{\text {sys }}$ in volts & Maximum input current, $I_{\text {inv }}(\max )$ in amps \\
Voltage at maximum power, $E_{\mathrm{STC}}(\max )$ in volts & Nominal power output, $P_{\text {inv }}$ in $\mathrm{W}$ \\
Maximum power, $\left(P_{\text {array }}\right)_{\operatorname{maxp}}$ in $\mathrm{W}_{\mathrm{P}}$ & Conversion efficiency, $\eta_{\text {conv. }}$ in $\%$ \\
Temperature coefficient for open-circuit voltage, $T_{\mathrm{Eoc}}$ in $\%$ per ${ }^{\circ} \mathrm{C}$ & Nominal efficiency, $\eta_{\text {conv. }}$ in $\%$ \\
Temperature coefficient for maximum power voltage, $T_{\text {Emax }}$ in $\%$ per ${ }^{\circ} \mathrm{C}$ & \\
\hline \hline
\end{tabular}

By using the peak hour approach we can write:

$$
(\text { Energy })_{r}=\left(P_{r}\right)_{\mathrm{ac}}\left[\frac{S_{H}\left(\mathrm{kWh} / \mathrm{m}^{2} / \mathrm{yr}\right)}{S_{S}\left(\mathrm{~kW} / \mathrm{m}^{2}\right)}\right] \times 365 \frac{\text { days }}{\mathrm{yr}}
$$

and

$$
\left(P_{r}\right)_{\mathrm{ac}}=\eta_{\text {conv. }}\left(P_{\text {array }}\right)_{\mathrm{dc}}
$$

Hence:

$$
(\text { Energy })_{r}=\left(P_{\text {array }}\right)_{\mathrm{dc}} \eta_{\text {conv. }} \times 365\left(S_{H} / S_{S}\right)
$$

or

$$
\left(P_{\text {array }}\right)_{\mathrm{dc}}=\frac{(\text { Energy })_{r} S_{S}}{S_{H} \times 365 \eta_{\text {conv. }}}
$$

where $\left(P_{\text {array }}\right)_{\mathrm{dc}}$ is the dc output power of the proposed PV array in Watts, $\left(P_{r}\right)_{\mathrm{ac}}$ is the ac output power of the inverter in Watts, and $\eta_{\text {conv. }}$ is the conversion efficiency from dc to ac according to inverter efficiency, dc cabling, temperature impact and module mismatch (select $\eta_{\text {conv. }}=97 \%$ ).

Assuming $S_{H}$ to be equal to $5.64 \mathrm{kWh} / \mathrm{m}^{2} /$ day (Kuwait annual averaged SR) and $S_{s}$ (SR at PV cell level under STC) as $1 \mathrm{~kW} / \mathrm{m}^{2}[5,6]$ yields the estimated rated power, $\left(P_{\text {array }}\right)_{\mathrm{dc}}$, to be equal to approximately $100 \mathrm{MW}$.

The second step is to determine the expected output voltages from the PV array such as the maximum value of the $\mathrm{PV}$ module open-circuit voltage, $E_{\mathrm{oc}}(\max )$, the minimum voltage at maximum power of the $\mathrm{PV}$ module, $E_{\mathrm{PV}}(\min )$, and the maximum voltage at maximum power of the $\mathrm{PV}$ module, $E_{\mathrm{PV}}(\max )$. Therefore, the maximum voltage at maximum power of the PV module,

$$
E_{\mathrm{PV}}(\max )=E_{\mathrm{STC}}(\max )\left[1-T_{\mathrm{Emax}}\left(t_{\mathrm{cmin}}-t_{\mathrm{STC}}\right)\right]
$$

where $E_{\mathrm{STC}}(\max )$ is the voltage at maximum power of the PV module at STC in volts, $T_{\mathrm{Emax}}$ is the temperature coefficient for maximum power voltage in $\%$ per ${ }^{\circ} \mathrm{C}, t_{\mathrm{cmin}}$ is the recorded minimum effective cell temperature of the PV panel at site in ${ }^{\circ} \mathrm{C}\left(\right.$ set $\left.t_{\mathrm{cmin}}=21{ }^{\circ} \mathrm{C}\right)$, and $t_{\mathrm{STC}}$ is the module temperature at STC in ${ }^{\circ} \mathrm{C}$.

The minimum voltage at maximum power of the PV module is:

$$
E_{\mathrm{PV}}(\min )=E_{\mathrm{STC}}(\max )\left[1-T_{\mathrm{Emax}}\left(t_{\mathrm{cmax}}-t_{\mathrm{STC}}\right)\right]
$$

where $t_{\text {cmax }}$ is the recorded maximum effective cell temperature of the PV panel at site in ${ }^{\circ} \mathrm{C}$ (set $t_{\mathrm{cmax}}=$ $\left.78^{\circ} \mathrm{C}\right)$.

On the other hand, the maximum PV module open-circuit voltage is: 


$$
E_{\mathrm{oc}}(\max )=E_{\mathrm{oc}}\left[1-T_{\mathrm{Eoc}}\left(t_{\mathrm{cmin}}-t_{\mathrm{STC}}\right)\right]
$$

where $E_{\mathrm{oc}}$ is the open-circuit voltage of the module at STC, $T_{\mathrm{Eoc}}$ is the temperature coefficient for opencircuit voltage in $\%$ per ${ }^{\circ} \mathrm{C}$.

The maximum percentage allowable of voltage drop across the dc cables, as specified in the GCC (Gulf Council Countries) standard, is equal to 7.5\%. Applying this percentage, we can correct the voltage given by (6) using

$$
E_{\mathrm{PV}}(\min )_{f}=0,925 E_{\mathrm{PV}}(\min ) \text { in volts }
$$

Hence $E_{\mathrm{PV}}(\min )_{f}$ is the final minimum voltage at maximum power of the PV module.

\section{Inverter Sizing Using SAIMA}

There are three criteria that can be used to decide on the type and number of the inverters [7]: maximum admissible input voltage, $E_{\text {inv }}(\max )_{\text {ad }}$, minimum admissible input voltage, $E_{\text {inv }}(\min )_{\text {ad }}$ and the current compatibility. The design of inverters will impose how to wire the PV modules together. If the maximum input voltage of the inverter, $E_{\mathrm{inv}}(\max )_{\mathrm{ad}}$, is less than the PV output voltage, $E_{\mathrm{PV}}(\max )$, the inverter will be damaged. Moreover, as the PV voltages in series are added, the value of $E_{\text {inv }}(\max )_{\text {ad }}$ will therefore determine the maximum number of modules in series. As the currents are added when strings are in parallel, the value of the input current of the inverter, $I_{\text {inv }}$, will determine the maximum number of parallel strings. In order to determine the number of panels connected within the whole array, the value of the maximum output power of the PV array, $\left(P_{\text {array }}\right)_{\mathrm{dc}}$, should not exceed the maximum allowable power of the connected inverters. The SAIMA algorithm has been applied to the particular GCPV system so that all three criteria can be satisfied.

First, the admissible input voltage limits of the inverter, $E_{\text {inv }}(\max )_{\text {ad }}$ and $E_{\text {inv }}(\min )_{\mathrm{ad}}$, are calculated. The maximum admissible input voltage of the inverter, $E_{\text {inv }}(\max )_{\mathrm{ad}}$, can be computed using:

$$
E_{\text {inv }}(\max )_{a d}=\eta_{\text {inv }} E_{\text {inv }}(\max )
$$

where $E_{\text {inv }}(\max )$ is the maximum input voltage of the inverter in volts and $\eta_{\text {inv. }}$ is the nominal efficiency of the inverter (select $\eta_{\text {inv. }}=94 \%$ ), whereas the minimum admissible input voltage of the inverter, $E_{\text {inv }}(\min )_{\text {ad }}$, can be calculated using:

$$
E_{\text {inv }}(\min )_{\text {ad }}=\left(\eta_{\text {inv }}+\eta_{\text {Edrop }}\right) E_{\text {inv }}(\min )
$$

where $E_{\text {inv }}(\min )$ is the minimum input voltage of the inverter and $\eta_{\text {Edrop. }}$ is the maximum percentage allowable of voltage drop across the terminals of the inverter (select $\eta_{\text {Edrop. }}$ as equals to $14 \%$ ).

\section{Panel or Array Sizing Using SAIMA}

A GCPV system consists of a number of panels (an array), which are normally mounted on a horizontal surface. A small increase or decrease in the size of the solar PV system can result in substantial differences in the outputs and return on investment of the system. Once we know the size of the solar PV array we can use any leading enterprise resource planning software to calculate the output of the array and the whole system in $\mathrm{kWh}$ (kilowatt hours). These calculations take into consideration the number of series and parallel PV modules, output voltages and currents, size of the inverter and some other factors, such as the orientation of the collectors and the percentage of the shaded area of the panels (over shading), to produce a conservative figure. Again, SAIMA can be implemented to compute the maximum number of parallel strings, $n_{p}$, as well as the maximum, $n_{s}(\max )$, and minimum, $n_{s}(\min )$, number of PV modules per string that satisfy the inverter voltage and current limits. These limits are subject to the following criteria: 
a. $n_{s}(\mathrm{mp})$ and $n_{s}(\mathrm{oc})$ are rounded down to the nearest integer,

b. $n_{s}(\mathrm{~min})$ is rounded up to the nearest integer,

c. $E_{\mathrm{PV}}(\max )<E_{\text {inv }}(\max )_{\mathrm{ad}}$ for both maximum power and open circuit conditions,

d. $E_{\mathrm{PV}}(\max )>E_{\mathrm{inv}}(\min )_{\mathrm{ad}}$ for both maximum power and open circuit conditions,

e. $n_{s}(\max )$ is the smaller value between $\mathrm{n}_{\mathrm{s}}(\mathrm{mp})$ and $n_{s}(\mathrm{oc})$,

f. $f_{o}$ is the oversize factor of PV module current ( $\operatorname{set} f_{o}=20 \%$ ),

where $n_{s}(\mathrm{mp})$ is the maximum number of PV modules per string based on voltage at maximum power of the PV module and $n_{s}(\mathrm{oc})$ is the maximum number of PV modules per string based on open circuit voltage of the PV module.

Consequently, the maximum number of parallel strings in the proposed PV array, $n_{p}$, will be computed as follows:

$$
n_{p}=\frac{I_{\mathrm{inv}}(\max )}{\left(1+f_{\mathrm{o}}\right) \times I_{\mathrm{SC}}}
$$

where $I_{\text {inv }}(\max )$ is the maximum input current of the inverter in amps and $I_{\mathrm{sc}}$ is the short-circuit current of the module at STC in amps.

A part from that, $n_{s}(\mathrm{mp})$ can be calculated using:

$$
n_{s}(\mathrm{mp})=\frac{E_{\mathrm{inv}}(\max )_{\mathrm{ad}}}{E_{\mathrm{PV}}(\max )}
$$

And $n_{s}(\mathrm{oc})$ can be determined using:

$$
n_{s}(\mathrm{oc})=\frac{E_{\mathrm{inv}}(\max )_{\mathrm{ad}}}{E_{\mathrm{oc}}(\max )}
$$

Besides that, $n_{s}(\mathrm{~min})$ is calculated as follows:

$$
n_{s}(\min )=\frac{E_{\mathrm{inv}}(\min )_{\mathrm{ad}}}{E_{\mathrm{PV}}(\min )_{f}}
$$

Now, the total number of calculated PV modules for the proposed PV array, $n_{t}(\mathrm{cal})$, can be determined as follows:

$$
n_{t}(\mathrm{cal})=n_{p} n_{s}
$$

where $n_{s}$ is the number of PV modules in series.

Moreover, the maximum calculated voltage of the array, $E_{\mathrm{cal}}$, is determined by:

$E_{\mathrm{cal}}=n_{s} E_{\mathrm{oc}}(\max )$ in volts

Andthe maximum calculated current of the array, $I_{\text {cal }}$, is found from:

$I_{\text {cal }}=n_{p} I_{\text {sc }}$ in amps

The PV array can take many configurations because $n_{s}$ may take two possibilities due to $n_{s}(\min )$ and $n_{s}(\max )$. Any PV array configuration will be discarded if it is not agreed with the following condition:

1) $n_{t}(\mathrm{cal})>n_{t}(\exp )$

2) $I_{\text {cal }}<I_{\text {inv }}(\max )$

3) $E_{\text {cal }}<E_{\text {sys }}$

where $n_{t}(\exp )$ is the total number of expected PV modules for the proposed system and it is calculated by:

$$
n_{t}(\exp )=\left(P_{\text {array }}\right)_{\mathrm{dc}} /\left(P_{\text {array }}\right)_{\operatorname{maxp}}
$$




\section{Important Sizing Parameters Calculated Using SAIMA}

There are some important parameters that may affect the sizing of the GCPV system, such as the actual capacity of the PV array, $\left(P_{\text {array }}\right)_{\text {act }}$, expected annual energy output in GWh/yr, (Energy) $)_{\text {exp }}$, inverter-to-PV array sizing factor, $\mathrm{Sf}\left(\mathrm{Sf}_{\min }<\mathrm{Sf}<\mathrm{Sf}_{\max }\right.$, for the GCC countries, $\mathrm{Sf}_{\max }$ is 0.85 and $\mathrm{Sf}_{\min }$ is 0.65$)$ and the excess factor, Ef. These values have to be calculated for all array configurations that were selected using the procedure detailed in the previous section. The following equations are used to compute the required sizing parameters.

$$
\begin{aligned}
& \left(P_{\text {array }}\right)_{\text {act }}=n_{t}(\mathrm{cal})\left(P_{\text {array }}\right)_{\operatorname{maxp}} \quad(\mathrm{MWp}) \\
& \mathrm{Sf}=P_{\text {inv }} /\left(P_{\text {array }}\right)_{\text {act }}
\end{aligned}
$$

where $P_{\text {inv }}$ is the nominal power output of the inverter, and:

$$
(\text { Energy })_{\text {exp }}=\left(P_{\text {array }}\right)_{\text {act }} \eta_{\text {conv. }} \times 365\left[S_{H} / S_{S}\right]
$$

After determining all these data, then the array configuration which yields the highest Sf will be selected as the final design solution. Thereafter, the area of one panel, $A_{\text {panel }}$, can be estimated using:

$$
A_{\text {panel }}=n_{t}(\mathrm{cal}) A_{m} \quad\left(\text { in } \mathrm{m}^{2}\right)
$$

where $A_{m}$ is the area of one PV module in $\mathrm{m}^{2}$.

\section{Results and Discussion}

The interactions between modules, inverters and the PV array have been explored and the sizing results of SAIMA have been investigated using different PV modules and inverters. The impact of the PV panel-layout is presented in Table 2. According to the input of SAIMA (five different types of both modules and inverters were used), it is clear that the combination of Type 3 (Sharp SKK-300 monocrystal SMC-PV module and Sunny Boy SB2500 inverter) has been chosen for the proposed GCPV system due to the highest value of Sf, which is approximately 0.8131 , with 43 PV modules in series in one string, $42 \mathrm{PV}$ modules in parallel in one panel and $132 \mathrm{PV}$ panels in the whole array. This suggests using that an array with the above configuration will achieve a maximum electrical energy of about 198.927 GWh/yr, an actual rated power output of $97.268 \mathrm{MW}$ covering an area of $0.5786814 \mathrm{~km}^{2}$.

Table 2. Sizing results using SAIMA

\begin{tabular}{ccccc}
\hline \multirow{2}{*}{ Results } & \multicolumn{3}{c}{ PV module and inverter combination } \\
\cline { 2 - 5 } & Type 1 & Type 2 & Type 3 & Type 4 \\
\hline PV modules in series, $n_{s}$ & 178 & 154 & 43 & 49 \\
PV modules in parallel, $n_{p}$ & 93 & 48 & 42 & 51 \\
Panel configuration $\left(n_{s} \times n_{p}\right)$ & $178 \times 93$ & $154 \times 48$ & $43 \times 42$ & $49 \times 51$ \\
Number of total panels & 47 & 83 & 132 & 67 \\
Number of iterations for inverter & 7 & 7 & 7 & 7 \\
Number of iterations for PV module & 3 & 8 & 11 & 14 \\
$P_{\text {array in } \mathrm{MWp}}$ Sf & 78.213 & 81.103 & 97.268 & 88.292 \\
$E_{\text {system }}(\mathrm{GWh})$ & 0.6218 & 0.7116 & 0.8131 & 0.7745 \\
$A_{\text {panel }}$ in $\mathrm{m}^{2}$ & 133.024 & 146.546 & 198.927 & 171.469 \\
& 15601.81 & 9440.32 & 4383.95 & 8619.34 \\
\hline
\end{tabular}

It is important that the maximum open-circuit voltage of the panel, as estimated by the proposed algorithm (SAIMA), does not exceed the highest dc voltage that the inverter can accept, which in this case is $3 \mathrm{kV}$. With 43 modules in series in one string, each having $E_{o c}$ at STC of $58.1-\mathrm{V}$, the string voltage could reach $43 \times 63.2=2717.6 \mathrm{~V}$. This is well below the $3 \mathrm{kV}$ limit. As shown in Fig. 2 and Fig. 3, the total efficiency of the system, $\eta_{\text {total }}$, has been achieved for each type of the combination selected. Although all types of combinations have produced efficiency of not less than 62\%, type (3) combination attained the highest efficiency of $91 \%$. 


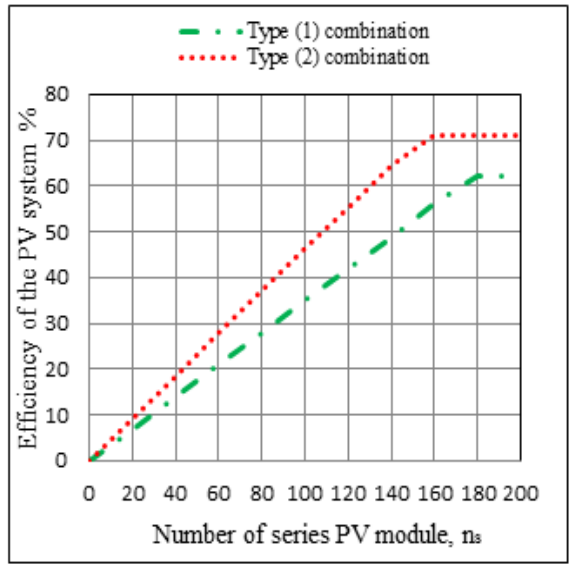

Fig. 2. Total efficiency of the PV system for Type (1) and (2) combinations.

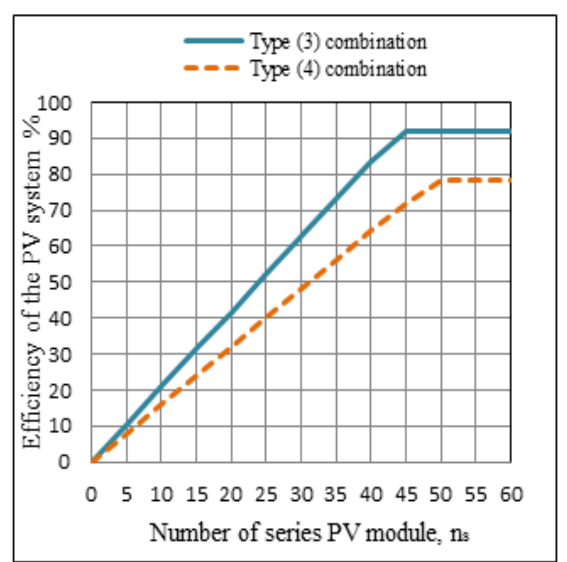

Fig. 3. Total efficiency of the PV system for Type (3) and (4) combinations.

SAIMA assumed the annual average SR, $S_{H}$, equal to $5.64 \mathrm{kWh} / \mathrm{m}^{2}$, but if average monthly SR were used for type 3 combination (for example) we would obtain the total power output of the proposed PV array as shown in Fig. 4. The output power, $\left(P_{\text {array }}\right)_{\text {act }}$, is varying between $35 \mathrm{MW}$, occurring between December and January, and 97.5 MW in June when it is at its maximum.

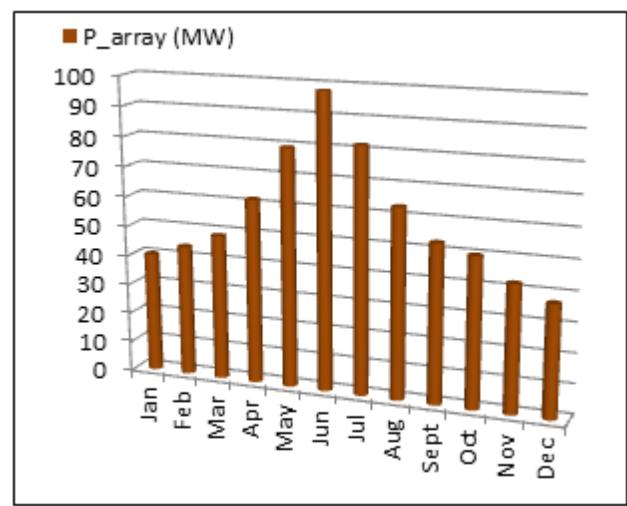

Fig. 4. Total power output of the proposed PV array at monthly averaged SR.

\section{Conclusions}

An iterative approach to the GCPV system sizing has been developed which gives a multicombination of array size and inverter capacity to meet the reliability requirement and the possibility to integrate the proposed GCPV system to Kuwait national grid. The conclusions of the study are summarized as follows:

(i) Monthly and yearly averaged SR profiles influence the size of the PV panel or array, as well as the inverter size, significantly.

(ii) SAIMA curves indicate the importance of the relationship between modules and inverters in the GCPV system. Different PV modules and inverters combinations on SAIMA curve have different array configuration $\left(n_{p} \times n_{s}\right)$.

(iii) The proposed GCPV system using SAIMA exhibits minimum system ratio of the generated energy by the system to the required energy.

(iv) Technical sizing outputs such as the number of PV modules, PV panel configuration and inverterto-PV panel sizing factor are the most important outputs of the proposed algorithm for the integration process to the grid. 


\section{References}

[1] Sulaiman SI, Rahman TKA, Musirin I. Novel intelligent sizing algorithm for grid-connected photovoltaic system design. International Review on Modeling and Simulations, 2010, 3(4):639-652.

[2] Sulaiman SI, Rahman TKA, Musirin I. Sizing grid-connected photovoltaic system. Presented at: IEEE Symposium on Industrial Electronics and Applications (ISIEA2011), Langkawi, 2011.

[3] Omar AM, Shaari S. Sizing verification of photovoltaic array and grid-connected inverter ratio for the Malaysian building integrated photovoltaic project. International Journal of Low-Carbon Technologies, 2009, 4(4):254-257.

[4] Muneer W. Large-scale solar PV investment planning studies. International Review on Modeling and Simulations, 2012, 2(6):449-456.

[5] Al-Enezi FQ, Sykulski JK, Ahmed NA. Visibility and potential of solar energy on horizontal surface at Kuwait area. Energy Procedia, 2011, 12:862-872. Also presented at: the IEEE International Conference on Smart Grid and Clean Energy Technologies, Chengdu, 2011.

[6] Al-Enezi FQ, Sykulski JK. Modelling of a photovoltaic module considering the solar energy available from horizontal surfaces over Kuwait area. Journal of Electronic Science and Technology, 2012, 10(2):173-180.

[7] Kjaer SB, Pedersen JK, Blaabjerg F. A review of single-phase grid-connected inverters for photovoltaic modules. IEEE Transactions on Industry Applications, 2005, 41(5):1292- 1306. 